\title{
Impact of Random Channel Mismatch on the SNR and SFDR of Time-interleaved ADCs
}

\author{
Gildas Leger, Eduardo J. Peralías, Adoración Rueda, Member, IEEE and José L. Huertas, Fellow, \\ IEEE
}

\begin{abstract}
Using several ADCs (Analog to Digital Converters) in parallel with convenient time offsets is considered as an efficient way to push the speed limits of data acquisition systems. However, a serious drawback of this time-interleaving technique is that any mismatch between the channels will damage the precision. This paper gives a probabilistic description of the problem, studying the impact of time skews, gain and offset mismatches. The Probability Density Function (PDF) of both Signal-to-Noise Ratio (SNR) and Spurious-Free-Dynamic Range (SFDR) are explicitly calculated, giving access to important statistical parameters. It is shown that the SNR and SFDR dispersion should not be neglected in making practical considerations for design decisions.
\end{abstract}

Index Terms-time-interleaving, parallel converters, ADC channel mismatch, Signal-to-Noise Ratio, Spurious-Free Dynamic Range.

\section{INTRODUCTION}

$\mathrm{M}$ ANY applications are requiring faster and faster analogto-digital converters. In RF design, for example, the trend is to move the analog to digital conversion to front-end or to intermediate frequency, in order to implement digitally the filtering and signal processing. Therefore, very high speeds are expected for the ADCs and literature reports up to 6bits at $1.3 \mathrm{GHz}$ [1]. However, these ADCs are usually flash or folding architectures, which are also resolution limited. Thus, it is becoming very difficult to go beyond these limits without losing too much precision or without using exotic and thus expansive technologies (HEMT in GaAs, optical or even superconducting ADCs) [2].

A possible way to gain some speed is to use the timeinterleaving technique, first presented by Black and Hodges [3]. Figure 1 shows a general sketch of the system. A number $M$ of medium-speed ADCs are operated in parallel but the sampling instants between two consecutive ADCs are shifted by a fraction $1 / M$ of their clock period. By multiplexing the channel output in a circular form, an equivalent high-speed $\mathrm{ADC}$ is obtained, that should have the same precision as the

Manuscript received September 30, 2003.

This work has been partially supported by the European Project SPRING $\mathrm{N}^{\mathrm{o}}$ IST-1999-12342.

The authors are with the Microelectronic Institute of Seville, 41012 Seville, Spain (phone: +34955056666; fax: +34955056686; e-mails: \{leger,peralias,rueda,huertas\}@imse.cnm.es). channel ADCs but with a speed $M$ times higher. The only fullspeed operation is the multiplexing, which is actually a digital operation. This technique trades area for speed in an approximately 1:1 ratio and can be used with almost any ADC architecture. Nevertheless, the drawbacks of this technique are two. One of them is that the ADC throughput and bandwidth are increased, but the latency will be the same as for the channel ADCs, which can be critical for some applications. But the most serious issue is that any mismatch between the channels will result in a resolution loss.

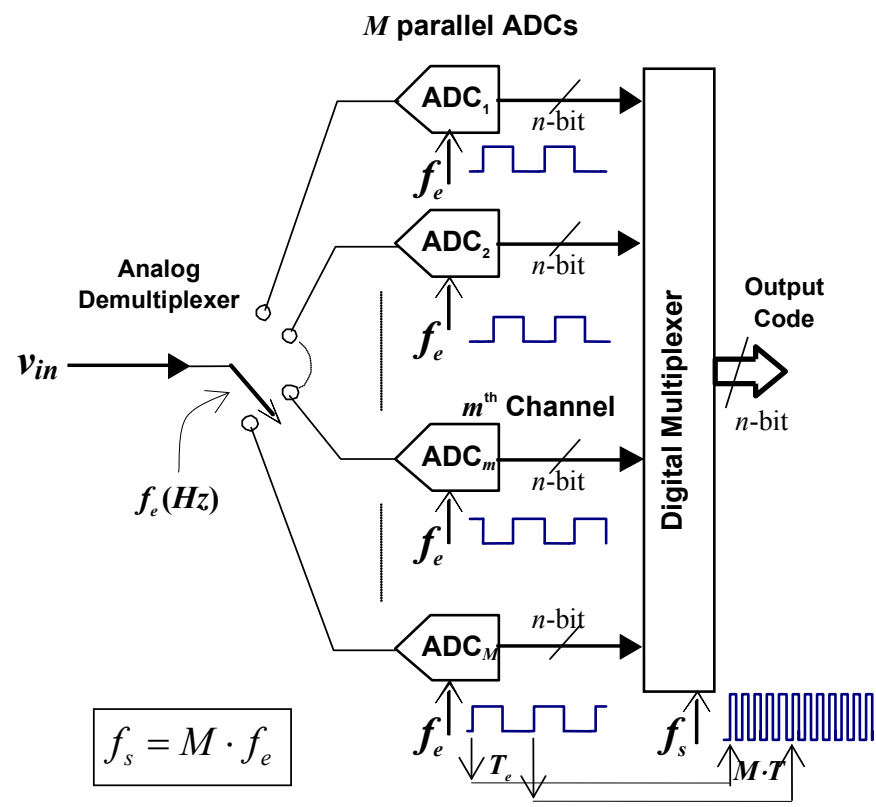

Fig. 1. Scheme of an $M$-channel time-interleaved ADC

Linearity mismatch will produce the appearance of noise spurs in the ADC output spectrum [4]. However, non-linearity is a common concern in ADC design and designers should manage to keep it low. Other sources of mismatch such as time skews in the sampling scheme will also degrade the performance as the signal will not be uniformly sampled. Random jitter has the same impact on the precision as for other ADCs, like flash ADCs or pipelined ADCs, and is not considered here. Time skews refer to systematic jitter between channels, which is specific to time-interleaved converters. Apart from time skews, offset and gain mismatches are also specially relevant. Indeed, for a single channel ADC, the exact value of gain or offset is not of much importance, as it will not affect the SNR or SFDR value. But for a time-interleaved 
architecture much more care should be taken as gain and offset mismatches will generate noise spurs in the signal band.

Estimations of the impact of these three kinds of mismatch on the SNR have already been proposed in other works [5]-[8] and even some solutions to cope with the mismatches have begun to appear in literature [9]-[11]. [5] settles the base of the spectrum study of non-uniformly sampled signals. Timeinterleaved $\mathrm{ADCs}$ are a direct application of this formalism and the author derives the expression of the expected SNR in the presence of time skews. [6] extends the study to the case of gain and offset mismatches and also derives an expression of the expected SNR for these mismatches. Reference [7] is also worth of mention as the authors take another approach and study the mismatch effect in the time domain. They are able to derive the SNR in the presence of the three kinds of mismatch simultaneously, but the expressions are available only for a 2channel and a 4-channel ADC. In [8], the multirate formalism is applied to time interleaved converters to study the impact of gain and offset mismatches. The authors make important statistical considerations but they do not bring these considerations down to the SNR. The work in this paper intends to give a deeper insight to the mismatch issue through a probabilistic treatment, completing the work presented in [12]. Closed expressions are developed for the probability density function of SNR and SFDR, as functions of the mismatch variance and the number of channels. The main interest to follow this probabilistic study is that it gives a more clear vision of the expected SNR or SFDR. Only a probabilistic model can provide precise information on the SNR dispersion. This practical information becomes of relevant importance for the establishment of safe design margins.

The paper is organized as follows. Section 2 introduces the basics of the spectral analysis of time interleaved ADCs. Section 3 derives a closed form for the SNR probability density function and validates it through simulations. Section 4 derives a closed form for the SFDR probability density function and validates it through simulations. Section 5 draws interesting information from the probabilistic model about the variation of the SNR and SFDR with the number of channels, it also shows how a designer could use the results of this study. Finally, Section 6 summarizes the conclusions of this paper.

\section{SPECTRAL ANALYSIS}

Shannon's theorem implies that an ADC running at frequency $f_{s}$ cannot convert signal of frequency $f_{\text {in }}$ above $f_{s} / 2$, the Nyquist frequency. Indeed, above $f_{s} / 2$, the input signal and its alias at frequency $f_{s}-f_{\text {in }}$ mix and cannot be separated. In a time-interleaved ADC, however, the aliases of all channel ADCs cancel each others when output signals are multiplexed, as it is illustrated in Fig. 2 for a 2-channel ADC. In this case, each elementary $\mathrm{ADC}$ is running at $f_{e}$, and after the interleaving the overall sampling frequency becomes $f_{s}=2 f_{e}$.

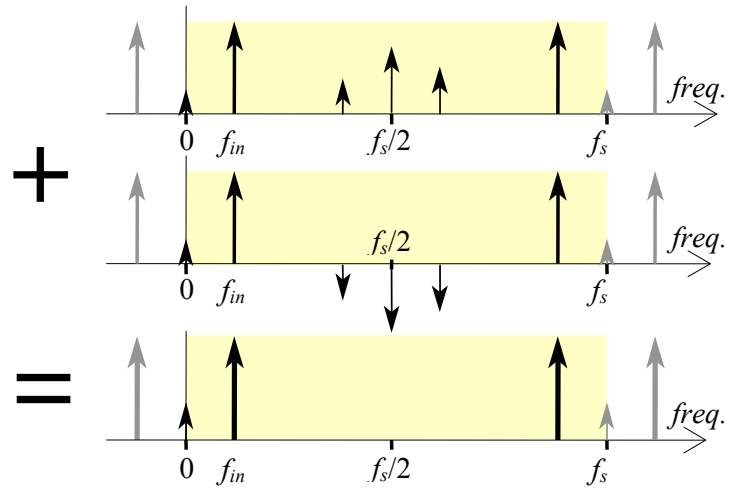

Fig. 2. Alias cancellation in an ideal 2-channel time interleaved ADC

However, in the presence of mismatches, alias cancellation is not complete and some spurs will remain on the final spectrum. For an $M$-channel ADC with offset mismatch, spurs will appear at every $M^{\text {th }}$ fraction of the sampling frequency (that is, $\left.f_{s} / M, 2 f_{s} / M, \ldots,(M-1) f_{s} / M\right)$. In the same way, for gain mismatch and time-skews, spurs will appear at the frequencies of the signal modulated by tones at every $M^{\text {th }}$ fraction of the sampling frequency (that is: $f_{s} / M+f_{i n}, f_{s} / M-f_{i n}, 2 f_{s} / M+f_{i n}, 2 f_{s} / M-$ $\left.f_{i n}, \ldots\right)$.

In that follows the same notation as in [5] and [6] is assumed, being:

$M$--- the number of interleaved channels

$T=1 / f_{s}$--- the equivalent sampling period of the ADC array

$t_{m}$--- the first real sampling instant of channel $m$, taking into account the time skews.

$t_{m}=(m-1) T-r_{m} T$, where $r_{m}$ is the time skew associated to channel $m$, expressed as a fraction of the sampling period.

Only the errors due to gain mismatch, offset mismatch and time skews will be considered. Hence, the channel ADCs are modeled in the simplest way possible as a fixed delay followed by a perfectly linear device with variable slope and offset. Figure. 3 presents the z-domain model of an M-channel time interleaved $\mathrm{ADC}$ with the three discussed mismatches

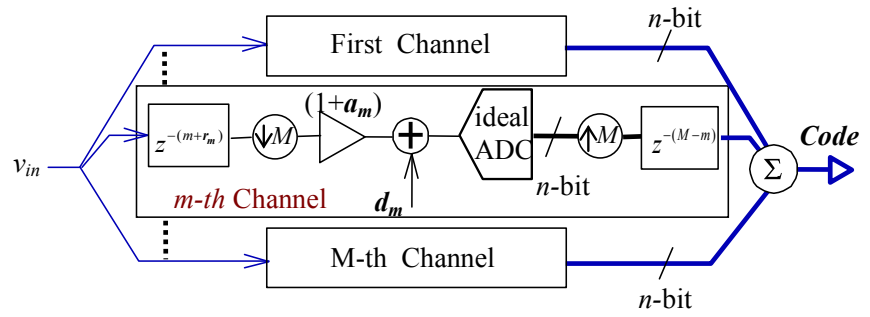

Fig. 3. Discrete-time model for a parallel M-channel interleaved ADC

Gain errors are introduced multiplying the signal amplitude by a channel-dependent term, $1+a_{\mathrm{m}}$, while offset errors are considered with an additional term, $d_{\mathrm{m}}$. These three nonidealities refer to mismatches due to the fabrication process and are assumed to be constant over time. That is an important point to ensure the validity of the Fourier transform that will be used in the following. 
Working in the z-domain and considering the time-interleaved output sequence $\left.\{T I-A D C(n)\}\right|_{n=0} ^{\infty}$ as the sum of the upsampled sequences $T I_{m}$ of every channel, ${ }^{1}$

$$
\begin{aligned}
& \left.\{T I-A D C(n)\}\right|_{n=0} ^{\infty}=\left(T I_{1}+T I_{2}+\ldots+T I_{M}\right)= \\
& +\left\{[\underbrace{A D C_{1}(1) 00 \ldots 0}_{(M)}, \underbrace{A D C_{1}(2) 00 \ldots 0}_{(M)}, \ldots, \underbrace{A D C_{1}(M) 00 \ldots 0}_{(M)}, \ldots]\right. \\
& +[\underbrace{0 A D C_{2}(1) 0 \ldots 0}_{(M)}, \underbrace{0 A D C_{2}(2) 0 \ldots 0}_{(M)}, \ldots, \underbrace{0 A D C_{2}(M) 0 \ldots 0}_{(M)}, \ldots]+\ldots \\
& \left.+[\underbrace{00 \ldots 0 A D C_{M}(1)}_{(M)}, \underbrace{00 \ldots 0 A D C_{M}(2)}_{(M)}, \ldots, \underbrace{00 \ldots 0 A D C_{M}(M)}_{(M)}, \ldots]\right\}
\end{aligned}
$$

the authors in [5] and [6] obtain a general expression of the digital output spectrum that for the particular case of a sinusoidal input with $\omega_{i n}$ frequency and $A_{0}$ amplitude, within the fundamental band $\left[0, f_{s}[\right.$, is

$$
\begin{aligned}
& F(\omega)=\frac{1}{M T} \sum_{k=0}^{M-1} e^{\frac{2 \pi k}{M}} \cdot\left\{\left[\sum_{m=1}^{M}\left(2 \pi d_{m} \cdot e^{-j k m \frac{2 \pi}{M}}\right)\right] \cdot \delta\left(\omega-k \frac{2 \pi f_{s}}{M}\right)+\right. \\
& +\left[\sum_{m=1}^{M}\left(\pi A_{0}\left(1+a_{m}\right) e^{-j \omega_{i n} r_{m} T} \cdot e^{-j k m \frac{2 \pi}{M}}\right)\right] \cdot \delta\left(\omega-\left(k \frac{2 \pi f_{s}}{M}+\omega_{i n}\right)\right)+ \\
& \left.+\left[\sum_{m=1}^{M}\left(\pi A_{0}\left(1+a_{m}\right) e^{+j \omega_{i n} r_{m} T} \cdot e^{-j k m \frac{2 \pi}{M}}\right)\right] \cdot \delta\left(\omega-\left((M-k) \frac{2 \pi f_{s}}{M}\right)-\omega_{i n}\right)\right\}
\end{aligned}
$$

As stated before, we can see that the last two terms of (1) stand for spurs appearing as uncanceled alias of the signal around the $M^{\text {th }}$ fractions of the sampling frequency. These two terms are due to both gain mismatch and time-skews. If only gain mismatches are considered, the magnitudes of these spurs are independent of the input signal frequency. This is not the case if only the time skews are considered as the term $r_{m}$ is multiplied by $\omega_{i n}$, the input signal frequency. The faster the signal, the more important the error due to imprecise sampling. The first term of (1) stands for tones that appear at the $M^{\text {th }}$ fractions of the sampling frequency and that are due to offset mismatch.

The output spectrum expressed in (1) does not take into account the quantization error. But this is not important because if the mismatch errors are as small as the quantization error, the overall $\mathrm{ADC}$ can be considered almost ideal. If mismatches are kept small, signal power can be written as

$$
P_{\text {signal }} \cong A_{0}^{2} / 2 \text {. }
$$

Equation (1) is graphically represented for the case of a 3channel ADC, in Fig. 4. Every spectral line is drawn together with its corresponding $k$ index as it is used in (1). Index $k=0$ is associated to the desired output signal.

\footnotetext{
1 The output sequence has the following general rule $T I-A D C\left(n_{m} \cdot M+m-1\right)=A D C_{m}\left(n_{m}\right)$ with $m \in\{1, \ldots, M\}$ the channel index and $n_{m} \geq 0$ the sample index of the $m^{\text {th }}$ channel.
}

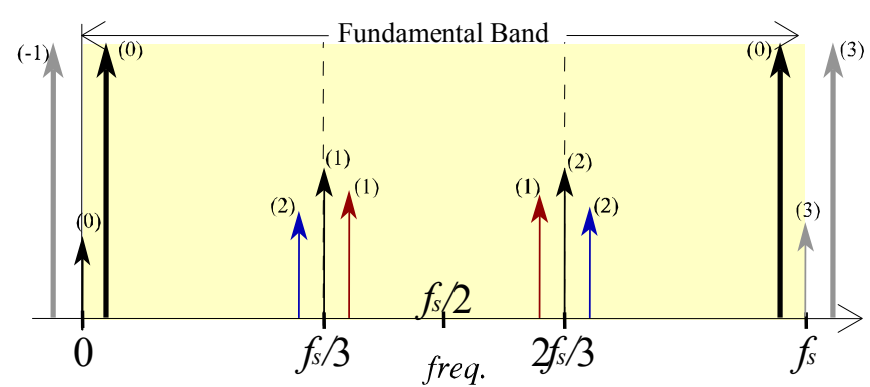

Fig. 4. A spectrum example for a 3-channel interleaved ADC

\section{PROBABILITY DENSITY FUNCTION FOR THE SNR}

To introduce the probability treatment, it will be assumed that all $a_{m}, d_{m}$ and $r_{m}$ in (1) are random variables distributed with a centered Normal law ${ }^{2}, x_{m} \sim N\left(0 ; \sigma_{x}\right)$, where $\sigma_{\mathrm{x}}$ is the interchannel standard deviation of the mismatch $x$ (that stands for either $a, d$ or $r$ ). These random variables can be normalized (standardized) such that

$$
x_{m}=\sigma_{x} x_{m}^{\prime} \text { with } x_{m}^{\prime} \sim N(0 ; 1)
$$

In the following development we will consider three initial assumptions:

- one kind of mismatch will probably dominate over the others. So, only one kind of mismatch will be studied at a time. This assumption will be commented at the end of this section.

- small time skews, which will allow the use of first order Taylor expansion,

$$
e^{-j \omega_{i n} r_{m} T} \approx 1-j \omega_{i n} T r_{m}=1-j 2 \pi \frac{f_{i n}}{f_{s}} \sigma_{r} r_{m}^{\prime}
$$

- the first spur due to the offset mismatch, appearing at $f=0$, is considered as the whole ADC offset.

The procedure to carry out the probabilistic approach is illustrated in Fig 5.

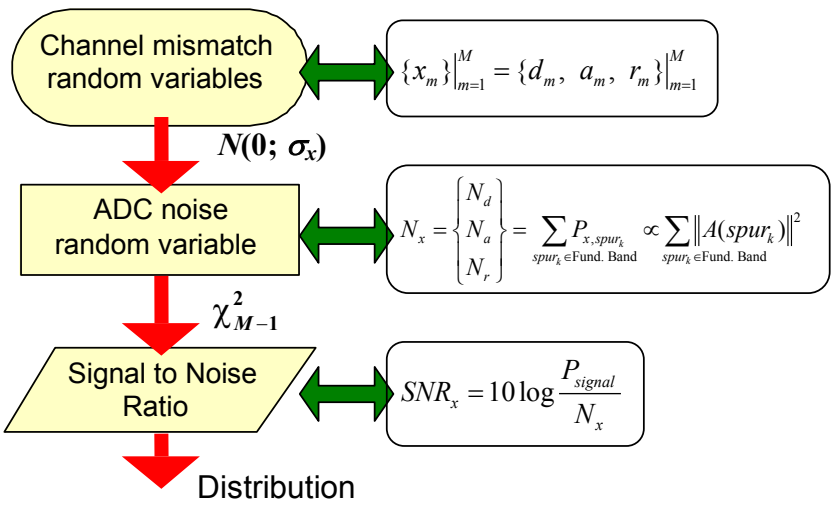

Fig. 5. Procedure leading to the SNR probability density function

The objective of the treatment is to get a closed form for the SNR PDF (Probability Density Function of the SNR). This is

2 A Normal Law is chosen as it is probably the most conservative assumption if no information on the technology mismatch is available. Moreover, at the ADCs level, the offset and gain mismatches as well as the time-skews can arise from lots of component mismatches. Then, to some extent, we can apply the Central Limit Theorem. 
in principle possible, because the SNR can be seen as a random variable that is a function of other random variables with known distributions (the channel mismatches). However, such a calculation is not straightforward and an intermediate step will be taken by calculating the noise power. It will come out that this intermediate random variable has a well-known distribution that will allow the calculation of the SNR PDF.

The first step is to get an expression of the noise power generated by the channel mismatches (see Fig. 5). The noise term corresponding to the offset mismatch will be the sum of all the spurs power at the $M^{\text {th }}$ fractions of the sampling frequency. In the digital output spectrum, all the offset spurs correspond to the first term of (1). Then, the noise power can be written

$$
N_{d}=\frac{1}{4 \pi^{2} f_{s}^{2}} \frac{1}{(M T)^{2}} \sum_{k=1}^{M-1}\left\|\sum_{m=1}^{M}\left(2 \pi d_{m} \cdot e^{-j k m \frac{2 \pi}{M}}\right)\right\|^{2} .
$$

Notice that the sum begins with $k=1$ in order to discard the spur at $f=0$, as stated before. After some simple developments (see Appendix A),

$$
N_{d}=\left\langle d_{m}^{2}\right\rangle-\left\langle d_{m}\right\rangle^{2}=\frac{M-1}{M} \cdot \sigma_{d}^{2} s_{d}
$$

where $\langle\cdot\rangle$ is the interchannel sample mean operator,

$$
\langle\cdot\rangle=\sum_{m=1}^{M}(.) / M
$$

and the variable $s_{d}$ is the interchannel sample variance of the offset mismatch defined as

$$
s_{x}=\sum_{m=1}^{M}\left(x_{m}^{\prime}-\left\langle x_{m}^{\prime}\right\rangle\right)^{2} /(M-1) .
$$

In the same way, the noise term arising from a gain mismatch can be extracted by making $r_{m}=0$ in the second and third terms of (1). Then the noise power for gain mismatch results

$$
N_{a}=\frac{A_{0}^{2}}{2} \cdot\left[\left\langle\left(1+a_{m}\right)^{2}\right\rangle-\left\langle 1+a_{m}\right\rangle^{2}\right]=\frac{M-1}{M} \cdot \frac{A_{0}^{2}}{2} \cdot \sigma_{a}^{2} s_{a} .
$$

Finally, if $r_{m}$ is small, which should be granted by design, the exponential term can be linearly expanded and the noise term for time skews becomes

$$
\begin{aligned}
N_{r} & =\frac{A_{0}^{2}}{2} \cdot\left[\left\langle\left\|1-j \omega_{i n} T r_{m}\right\|^{2}\right\rangle-\left\|\left\langle 1-j \omega_{i n} T r_{m}\right\rangle\right\|^{2}\right]=\ldots \\
& =\frac{M-1}{M} \cdot \frac{A_{0}^{2} \omega_{i n}^{2} T^{2}}{2} \cdot \sigma_{r}^{2} s_{r} .
\end{aligned}
$$

It is easily seen that all the noise terms have the same form with respect to the random variables. Then, the probability treatment will be the same for all of them and only the first coefficient will make the difference between the different mismatches.

The next step consists in finding the distribution of the noise power (see Fig. 5), which means evaluating the PDF of the interchannel sample variance $s_{x}$.

Here, the key point is to recognize that, as all the $x_{m}^{\prime}$ are distributed as $N(0 ; 1)$, then the sample variance $s_{x}$ is distributed like a well-known Chi-square law with $M-1$ degrees of freedom [13],

$$
s_{x} \sim \chi_{M-1}^{2}(s)=\left.\frac{1}{2^{v / 2} \Gamma(v / 2)} s^{(v / 2)-1} e^{-s / 2}\right|_{v=M-1} \quad, s>0
$$

where $\Gamma$ is the gamma function.

Now that the PDF of the noise power is known, it is possible to deduce the PDF of the Signal-to-Noise Ratio (see Fig. 5). The general expression for any mismatch would be,

$$
S N R_{x}=10 \log \left(\frac{P_{\text {signal }}}{N_{x}}\right)=10 \log \left(\frac{M \lambda_{x}}{M-1} \cdot \frac{1}{S_{x}}\right)=F_{x}\left(s_{x}\right)
$$

where applies

$\lambda_{d}=A_{0}^{2} /\left(2 \sigma_{d}^{2}\right)$ for the offset mismatch,

$\lambda_{a}=1 / \sigma_{a}^{2}$ for the gain mismatch and

$\lambda_{r}=\left[f_{s} /\left(2 f_{\text {in }}\right)\right]^{2} /\left(\pi^{2} \sigma_{r}^{2}\right)$ for the time-skews.

In (12), the function $\left(F_{x}\right)$ relating the random variable $S N R_{x}$ to the random variable $s_{x}$ is a bijection. Therefore, it is possible to go a step forward and calculate the explicit probability density function of the SNR using the following relation for a variable change [13],

$$
\begin{aligned}
& \text { if } \operatorname{SNR}_{x}=F_{x}\left(s_{x}\right) \text { and } s_{x} \sim \chi_{M-1}^{2} \text {, then } \\
& \begin{aligned}
S N R_{x} & \sim h_{S N R_{x}}(\xi)=\int_{\mathrm{R}} d s \cdot \chi_{M-1}^{2}(s) \cdot \delta\left(\xi-F_{x}(s)\right)=\ldots \\
& =\chi_{M-1}^{2}\left(F_{x}^{-1}(\xi)\right) \cdot\left|\frac{d}{d z}\left(F_{x}^{-1}\right)\right|_{z=\xi} \quad, \xi>0
\end{aligned}
\end{aligned}
$$

Using (12), it comes

$$
h_{S N R}\left(S N R_{x}\right)=\left.\frac{\alpha \cdot(v / 2)^{v / 2}}{\Gamma(v / 2)} e^{-\frac{v}{2} \cdot\left(u+e^{-u}\right)}\right|_{u=\alpha \cdot\left(S N R_{x}-S N R_{x}\right)}
$$

with

$$
\begin{aligned}
& \alpha=\ln 10 / 10 \quad, v=M-1 \quad \text { and } \\
& S \hat{N} R_{x}=10 \log \left(\frac{M \lambda_{x}}{M-1}\right) .
\end{aligned}
$$

Now, the final expression (17) will allow to make more direct computations and estimate confidence intervals for the SNR. Notice that the obtained standardized expression is common to the three types of mismatch, which means that their impact will be the same on the SNR. The only difference resides in the scaling parameters $\lambda_{\mathrm{x}}$ that are taken into account in (17) through the term $S \hat{N} R_{x}$ given by (18). Actually, it is also possible to verify (differentiating) that this term is the most probable value of the SNR.

Regarding the SNR estimate for the offset mismatch provided in [6], it can be proved that it would match the SNRmost-probable value $S \hat{N} R_{x}$, if the first spur (at $k=0$ ) were not accounted as noise.

In order to verify the correctness of these probability density functions, the calculated results were compared to 2000 Monte Carlo simulations of a virtual 14-bit M-time interleaved ADC. The time-interleaved converter was modeled in MATLAB as an array of ideal converters and the non-idealities were introduced at the front-end of each channel (see Fig. 3). For each run, $M$ random mismatch were sampled from a $N\left(0 ; \sigma_{x}\right)$ law and applied to the channel ADCs. The FFT of the 
distorted sine-wave output was then obtained and the SNR was evaluated.

Figure 6 shows the results corresponding to the offset mismatch. The $S N R_{d}$ does spread over the distribution predicted by the mathematical model (17).

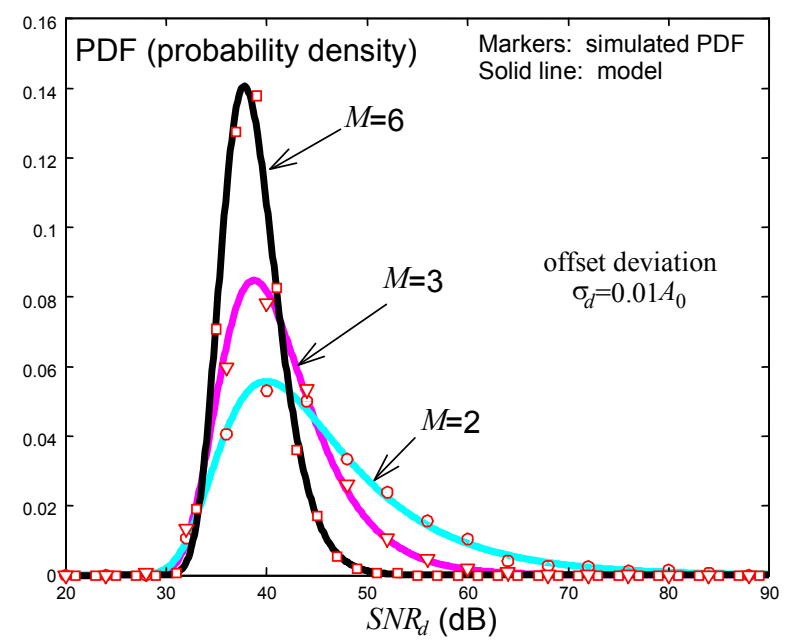

Fig. 6. PDF for an offset mismatch of $\sigma_{d}=0.01$, with $A_{0}=1$

In the same way, Fig. 7 represents the variation of the PDF for different time-skews in the case of $M=4$. The matching between the model and the simulation is once again very convincing. It can be noticed from Fig. 7 that the dispersion does not scale with the mismatch amplitude. The gain mismatches would provide the same kind of curves. Extensive simulations have been carried out to cover a wide range of cases but are not presented here for the sake of brevity. Indeed, (17) shows that the PDFs have the same normalized form for the three kinds of mismatch.

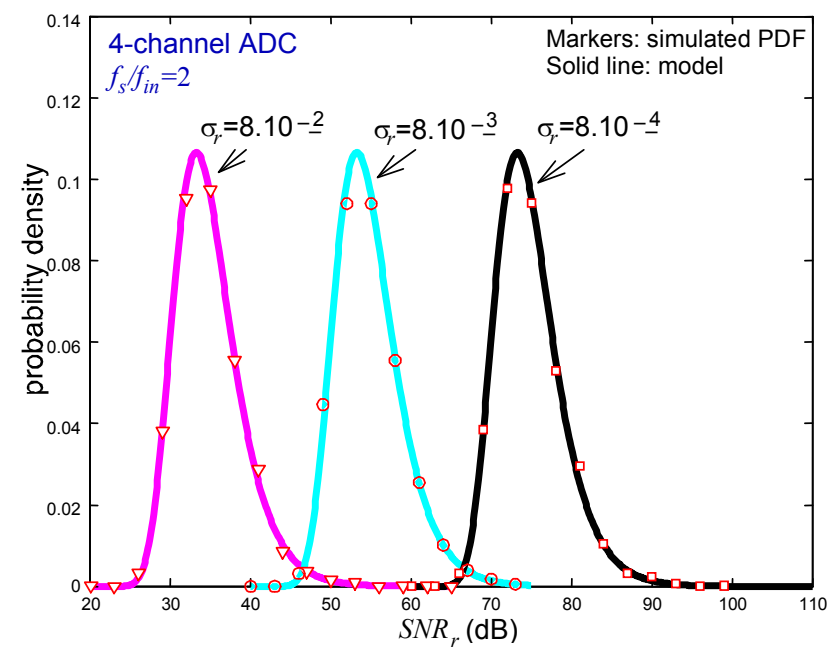

Fig. 7. $S N R_{r}$ PDF for $M=4$ and different time-skew deviations

At the beginning of this section, it was assumed that one kind of mismatch would dominate over the others. Nevertheless, it is legitimate to wonder what can happen if this is not the case. Actually, it is possible to follow the reasoning presented in this section without making the approximation of only one mismatch at a time. It can easily be shown that the total noise comes to be the sum of the three noise contributions already calculated, $N_{t o t}=N_{a}+N_{r}+N_{d}$. However, it becomes very difficult to extract the close form of the probability density function of such a sum. Indeed, what has to be done is to calculate the convolution product of three weighted Chi-square functions. This problem has no simple solution unless the coefficients of the variables are the same, that is, $\lambda_{\mathrm{a}}=\lambda_{\mathrm{r}}=\lambda_{\mathrm{d}}$ (see (13) to (15)). In that case, it could be shown that the resulting variable would follow a Chi-square law with $3(M-1)$ degrees of freedom and weight $3 / \lambda$ instead of $(M-1)$ degrees of freedom and weight $1 / \lambda$. Similarly, if two of the three contributions are of the same order an the third has a much lower weight, the sum would follow a Chi-square law with $2(M-1)$ degrees of freedom and weight $2 / \lambda$.

\section{Probability Density Function For the SFDR}

Figure 8 shows the flow of the procedure to obtain the PDF of the Spurious-Free Dynamic Range (SFDR). The reasoning is very similar to the SNR case and, once again, we will follow the same steps for the 3 types of mismatch. The first step consists in calculating the PDF of the power of $k^{\text {th }}$ spur which a priori should depend on the value of $k$ that defines the spur position in the spectrum. The second step consists in getting the PDF of the power maximum, and the last step finally deduces the PDF of the SFDR.

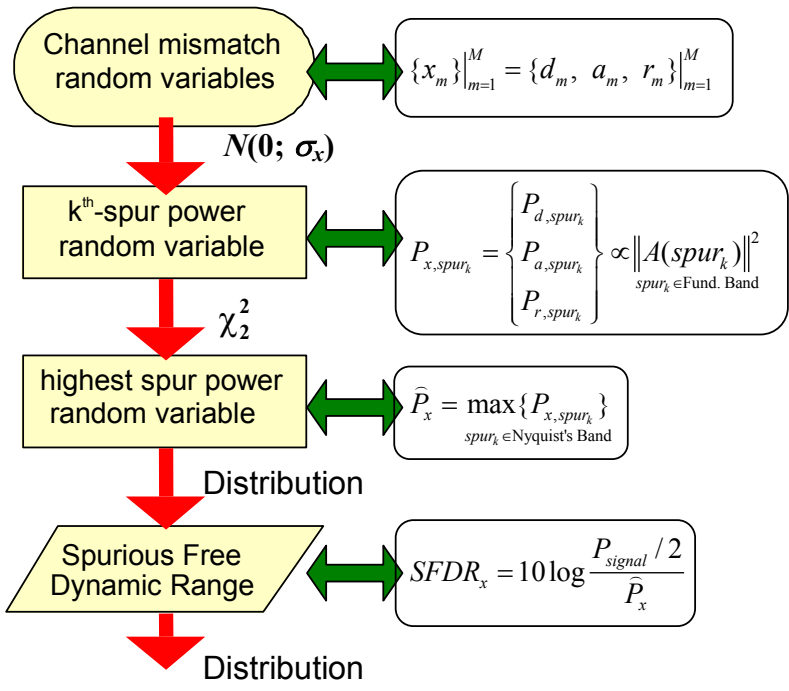

Fig. 8. Procedure leading to the SFDR probability density function

According to the flow presented in Fig. 8, the first task is to calculate an expression of the power density of a spur due to the offset mismatch,

$P_{d}^{\text {spur }}(k)=\frac{1}{M^{2}}\left\|\sum_{m=1}^{M}\left(d_{m} \cdot e^{-j n m \frac{2 \pi}{M}}\right)\right\|^{2}=\frac{1}{M^{2}} \cdot \sigma_{d}^{2} S_{d}(k), k \in[1, M-1]$.

For the gain mismatch the result is very similar.

$$
\begin{aligned}
P_{a}^{\text {spur }}(k) & =\frac{A_{0}^{2}}{4 M^{2}}\left\|\sum_{m=1}^{M}\left(1+a_{m}\right) \cdot e^{-j n m \frac{2 \pi}{M}}\right\|^{2}= \\
& =\frac{A_{0}^{2}}{4 M^{2}} \cdot \sigma_{a}^{2} S_{a}(k) \quad, k \in[1, M-1] .
\end{aligned}
$$

For the time-skews, the orders higher than the first in the 
Taylor development of (4) have been neglected,

$$
\begin{aligned}
P_{r}^{\text {spur }}(k) & =\frac{A_{0}^{2}}{4 M^{2}}\left\|\sum_{m=1}^{M}\left(1-j r_{m} \omega_{i n} T\right) \cdot e^{-j n m \frac{2 \pi}{M}}\right\|^{2}= \\
& =\frac{A_{0}^{2} \omega_{i n}^{2} T^{2}}{4 M^{2}} \cdot \sigma_{r}^{2} S_{r}(k) \quad, k \in[1, M-1] .
\end{aligned}
$$

It appears clearly that the probability treatment will be the same for the three mismatch contributions, as the term $S_{x}(k)$ has the same form in (19), (20) and (21). Therefore, one can treat the case of the offset mismatch and then take the results to the gain mismatch and time-skew cases. ${ }^{3}$

An equation relating the spur power to the mismatch random variables has been obtained, so the next step of the flow described in Fig. 8 consists in finding the probability density function of the spur power.

Notice that in (19), (20) and (21), another symmetry exists, $P_{x}^{\text {spur }}(k)=P_{x}^{\text {spur }}(M-k)$, therefore, hereinafter only the spurs corresponding to the integers $k$ comprised between 1 and $\lfloor M / 2\rfloor$ are going to be considered for SFDR calculation.

Let us consider the case $M=4$. The first spur at $k=1$ is computed as the sum of the channels offset over the $4^{\text {th }}$-order roots of unity (see Fig. 9a). For $k=3$, the spur power is the same as for $k=1$. However, for $k=2$, the sum is performed over the square roots of unity in only one dimension of the phase plane.

For $k=1$. the power would behave as

$$
\left.S_{d}(k=1)\right|_{M=4}=\left(d_{4}^{\prime}-d_{2}^{\prime}\right)^{2}+\left(d_{3}^{\prime}-d_{1}^{\prime}\right)^{2}
$$

But for $k=2$, it would behave as

$$
\left.S_{d}(k=2)\right|_{M=4}=\left(d_{4}^{\prime}+d_{2}^{\prime}-d_{3}^{\prime}-d_{1}^{\prime}\right)^{2}
$$

For other values of $M$ (see Fig. $9 \mathrm{~b}$ for the case of $M=5$ ), the same phenomenon can be observed.

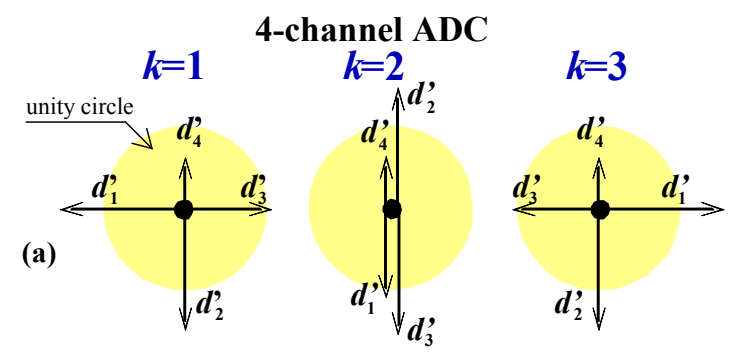

5-channel ADC

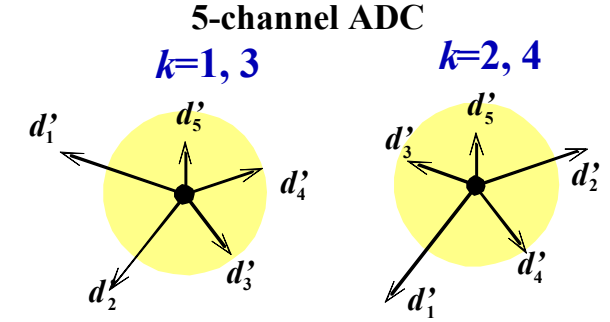

Fig. 9. a) Spur generation as function of index $k$ for $M=4$ (even) channels b) Spur generation as function of index $k$ for $M=5$ (odd) channels

If $k$ is different from $M / 2$ (which is always the case when $M$ is odd) the offset contributions should be projected on the two

3 The relationship between the variable $S_{x}(k)$ and the sample variance defined in (8) is $s_{x}=(1 / M(M-1)) \sum_{k=1}^{M-1} S_{x}(k)$. dimensions of the phase plane and all the spurs would have the same type of PDF, but for the spur in $k=M / 2$ with $M$ even, the PDF would be different because all the offset contributions project on the same dimension. This demonstration is presented in the Appendix B.

Expanding (22) and (23) for $k$ different from $M / 2$, it results,

$$
\left.S_{x}(k)\right|_{k \neq \frac{M}{2}} \Leftrightarrow\left(\sum_{m=1}^{\lfloor\mathrm{M} / 2\rfloor} x_{m}^{\prime}\right)^{2}+\left(\sum_{m=1+\lfloor\mathrm{M} / 2\rfloor}^{M} x_{m}^{\prime}\right)^{2}
$$

and for $k=M / 2$ with $M$ even,

$$
\left.S_{x}(k)\right|_{k=\frac{M}{2}} \Leftrightarrow\left(\sum_{m=1}^{M} x_{m}^{\prime}\right)^{2}
$$

The sum of $n$ variables distributed as a Normal law $N(0 ; 1)$ is distributed as a Normal law $N(0 ; \sqrt{n})$, and the sum of $v$ squared variables distributed as $N(0 ; 1)$ is distributed as a Chisquare law with $v$ degrees of freedom [13]. So (24) and (25) lead to

$$
\begin{gathered}
\left.S_{x}(k)\right|_{k \neq \frac{M}{2}} \sim h_{o}(s)=\frac{2}{M} \chi_{2}^{2}\left(\frac{2 s}{M}\right)=\frac{e^{-s / M}}{M}, s>0 \\
\left.S_{x}(k)\right|_{k=\frac{M}{2}} \sim h_{e}(s)=\frac{1}{M} \chi_{1}^{2}\left(\frac{s}{M}\right)=\frac{e^{-s /(2 M)}}{\sqrt{2 \pi M s}}, s>0
\end{gathered}
$$

The next step to obtain the SFDR PDF is to get the PDF of the maximum power density,

$$
\hat{P}_{x}^{\text {spur }}=\max _{k}\left\{P_{x}^{\text {spur }}(k)\right\}, k \in\{1,2, \ldots,\lfloor M / 2\rfloor\} .
$$

The similarities in the relationships (19), (20) and (21) justify that it is only necessary to find the PDF of the $\widehat{S}_{x}=\max _{k}\left\{S_{x}(k)\right\}$.

As stated in [13], the Cumulative Density Function (CDF) of the maximum of a set of independent random variables is the product of their individual CDFs. Although it is not obvious that the spurs are statistically independent, the fact that for different spurs the offset contributions are summed in a different order compensates the fact that the contributions are actually the same (see Fig. 9b) and therefore, produces an independent statistic behavior (the exact demonstration is made in the Appendix C).

The PDF of the maximum becomes (differentiating the CDFs product):

- if $M$ is odd, all the $(M-1) / 2$ spurs have the distribution of (26),

$$
\widehat{S}_{o x}=\left.\max _{k}\left\{S_{x}(k)\right\}\right|_{M \text { odd }} \sim h_{o}^{\max }(s)=\frac{M-1}{2}\left(H_{o}(s)\right)^{\frac{M}{2}-1} \cdot h_{o}(s) \text { (28) }
$$

- if $M$ is even, $(M / 2-1)$ spurs behave as (26) and the spur at $k=M / 2$ behaves as (27),

$$
\begin{aligned}
\widehat{S}_{e x} & =\left.\max _{k}\left(S_{x}(k ; M)\right)\right|_{M \text { even }} \sim h_{e}^{\max }(s)=\ldots \\
& =\left(H_{o}(s)\right)^{\frac{M}{2}-2} \cdot\left[\left(\frac{M}{2}-1\right) H_{e}(s) h_{o}(s)+h_{e}(s) h_{o}(s)\right]
\end{aligned}
$$

The functions $H_{o}$ and $H_{e}$ are the integrals (the CDFs) of the 
PDFs $h_{o}$ and $h_{e}$ respectively,

$$
\begin{gathered}
H_{o}(s)=1-e^{-s / M} \quad, s>0 \\
H_{e}(s)=\operatorname{erf}\left(\sqrt{\frac{s}{2 M}}\right) \quad, s>0
\end{gathered}
$$

Finally, the last step to obtain the SFDR PDF is to realize the variable change using the SDFR definition in the same way that it was made in (16).

It should be considered that the power density of a sinusoidal signal in $f=f_{\text {in }}$ is $P_{\text {signal }} / 2 \approx A_{0}^{2} / 4$, then, the definition of the SFDR provides

$$
S F D R_{x}=10 \log \left[\frac{P_{\text {signal }} / 2}{\widehat{P}_{x}^{\text {spur }}}\right]=10 \log \left(\frac{M^{2} \lambda_{x}^{\prime}}{\widehat{S}_{x}}\right)=F_{x}^{\prime}\left(\widehat{S}_{x}\right)
$$

where $\lambda_{d}^{\prime}=\lambda_{d} / 2, \lambda_{a}^{\prime}=\lambda_{a}, \lambda_{r}^{\prime}=\lambda_{r}$ (see (13), (14), (15)).

Using (28), (29) and (32), the SFDR probability density function becomes:

- for $M$ odd,

$$
h_{S F D R}\left(S F D R_{x}\right)=\left.\alpha \frac{v(v+1)}{2} u \cdot e^{-u}\left(1-e^{-u}\right)^{\frac{v}{2}-1}\right|_{u=(v+1) \lambda_{x}^{\prime} e^{-\alpha S S F D R_{x}}}
$$

- for $M$ even,

$$
\begin{aligned}
h_{S F D R}\left(S F D R_{x}\right) & =\alpha(v+1) u\left(1-e^{-u}\right)^{v / 2} . \\
& {\left[\frac{v-1}{2(v+1)} e^{-u} \operatorname{erf}(\sqrt{u / 2})+\right.} \\
& +\left.\frac{1}{(v+1) \sqrt{2 \pi u}}\left(1-e^{-u}\right) e^{-u / 2}\right|_{u=(v+1) \lambda_{x}^{\prime} e^{-\alpha . S F D R_{x}}}
\end{aligned}
$$

where the coefficients $\alpha$ and $v$ were defined in (18).

The correctness of the derived PDFs is verified comparing the values obtained from the equations to 2000 Monte Carlo simulations. For each run, $M$ random offsets were sampled from a $N\left(0 ; \sigma_{d}\right)$ law and taken into account into the same ADC model that has been used in Section 3. The FFT of the distorted sine-wave output was then obtained and the SFDR was evaluated. Figure 10 presents a simulation for time skews with $\sigma_{r}=0.005$ and $f_{i n}=f_{s} / 2$. The matching between the model and the simulation is very good, for both $\mathrm{M}$ even and odd.

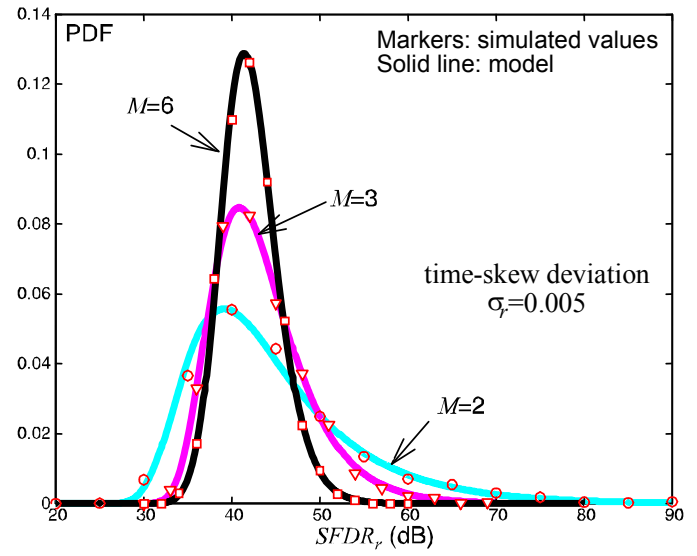

Fig. 10. SFDR PDF for a time-skew mismatch of $\sigma_{r}=0.005$

In Fig. 11, an ADC with 4 channels has been simulated for different values of time-skews. The results are also in good agreement with the prediction. Moreover we can notice that, as for the SNR, the dispersion does not depend on the amplitude of the time-skews. Simulations for offset mismatch and gain mismatch have also been realized and exhibit the same level of agreement with the model than the one presented here for time-skews. They are not presented for the sake of brevity.

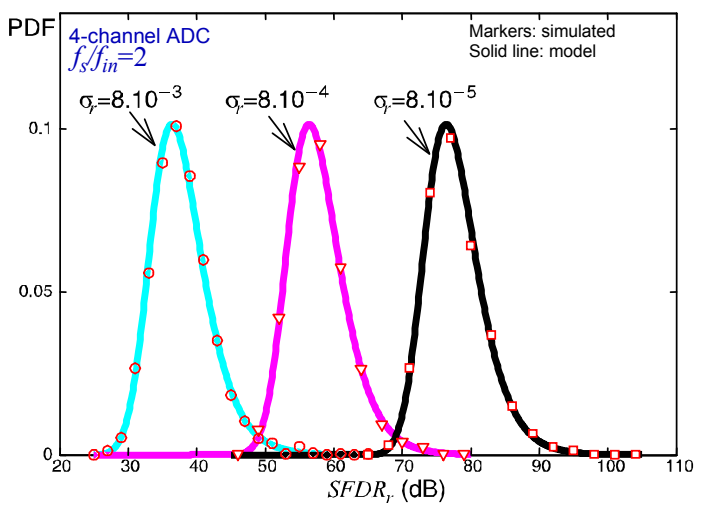

Fig. 11. SFDR probability density function for $M=4$, with different timeskew deviations

\section{RESULT DISCUSSION}

The mathematical model obtained through the probabilistic treatment described along this paper intends to provide a deeper and clearer understanding of the channel mismatch issue in time-interleaved ADCs. Various benefits can be drawn from this approach that could not be foreseen with previous simpler models. Indeed, in the same way that a measurement is meaningful only if its precision is known, a parameter estimate should always be provided together with a confidence interval or some other information on its dispersion. In Fig. 12, the variation of the dispersion in SNR due to offset mismatch is represented as function of the number of channels. As was stressed in Section 3, the SNR PDFs have the same form for the three kinds of mismatch. The only difference arises in the scaling of the $\lambda_{x}$ parameters (see (13) to (15)).

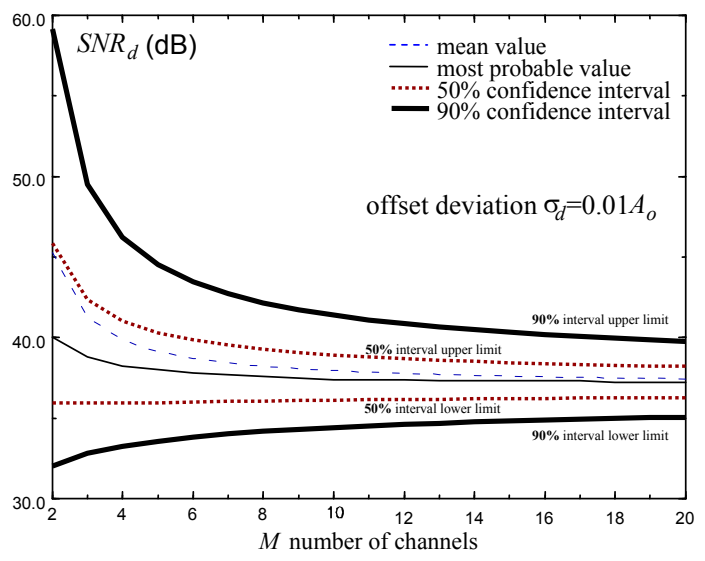

Fig. 12. Variation of the SNR dispersion with $M$, for an offset mismatch $\sigma_{d}=0.01 A_{\mathrm{o}}$

Therefore, the gain mismatch and time skews would follow the same trend as the offset mismatch in Fig. 12. It is worth 
noticing in Fig. 12 the strong dependence of the dispersion on $M$. It can be calculated that for $M=2$ the $90 \%$ confidence interval is as large as $27 \mathrm{~dB}$ (i.e. 4.5 effective bits), whereas for $M=8$ it reduces to only $8 \mathrm{~dB}$ (i.e. 1.3 effective bits).

Usually most designs are likely to implement a small number of channels in order to limit the chip area. But as can be seen in Fig. 12, for small values of $M$, the SNR dispersion is quite large, and this information should thus be taken into account when fixing the design goals. Therefore, the result given by the SNR estimate proposed in [6] reveals to be misleading, underestimating mismatch impact. In the same way, Fig. 13 shows the evolution of the expected SFDR together with its standard deviation. Here again the importance of the dispersion for small values of $M$ can be seen. Moreover, it could be interesting to comment two other issues. The first one is that the SFDR is actually increasing with the number of channels. When the number of channel is increased, more spurs appear in the signal band. The mean total noise, which has been seen to remain near constant with $M$ (see Fig. 12) is splitted between more and more spurs. As a result, the mean height of each spur decreases and the SFDR increases. This is quite intuitive as a spur is formed by an average of the rotated channel alias. As the channel contribution is zero mean, the average will tend to zero when $M$ tend to the infinite. However, this trend is not as significant as it could be expected (only $3 \mathrm{~dB}$ are gained from 2 to 8 channels). Indeed, the SFDR does not rely on the mean spur but on the highest spur. Therefore, the fact that the overall spur height decreases is somewhat compensated by the fact that there are more spurs, and thus more possibility to get a high spur. The other interesting issue is that even channel numbers give worse SFDR results than odd channel numbers. This is due to the fact that the spurs which correspond to $k=M / 2$ (for $M$ even), have a more dispersed height than the other, making them more likely to be the maximum spur, which determines the SFDR.

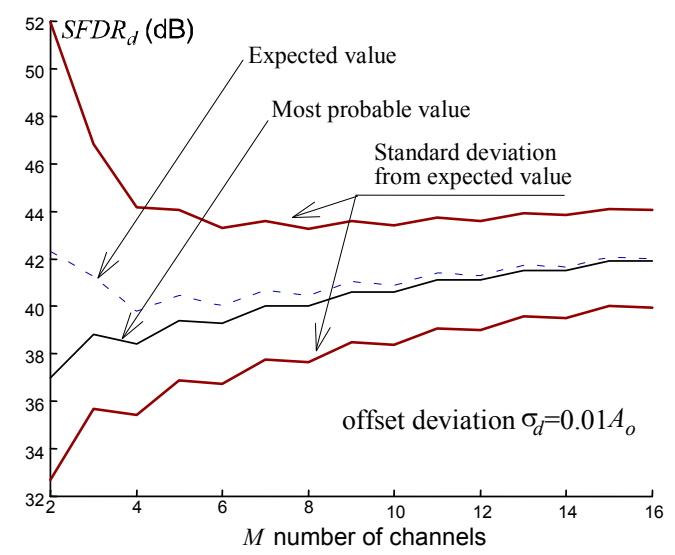

Fig. 13. SFDR prediction for an offset mismatch of $\sigma_{d}=0.01 A_{0}$

Therefore, it appears clearly that this mathematical model should help the designer to establish safe margins. A possible way to do this is evaluating the yield of a given design. Indeed, if the SNR design specification is, for instance, $60 \mathrm{~dB}$, the yield can be defined as the probability that the implemented ADC has an SNR greater than $60 \mathrm{~dB}$. That is

$$
Y=\int_{60}^{\infty} h_{S N R_{x}}(\xi) d \xi
$$

Based on (35), the Fig. 14 shows the evolution of the yield as function of the offset mismatch, for different $M$ values. The offset mismatch is expressed in Least Significant Bit (LSB) of a 10 bit ADC. It can be seen that, if the mismatch is as high as 1LSB, the 2-channel architecture is preferable as it will provide a yield of $68 \%$, versus a yield of only $22 \%$ for an 8 channel architecture. However, if mismatch of only 0.5LSB is managed, the trend is reversed and a 2-channel $\mathrm{ADC}$ will give a yield of $95.5 \%$ versus $97.5 \%$ for the 8 -channel ADC. This means that the high dispersion of the 2-channel architecture could be advantageous, as some ADCs will manage to reach the specification, but yield escapes could also be more likely. A point has been marked on Fig. 14 that is about common to all curves. It can be seen that for an offset mismatch above 0.6 LSB, the yield will be lower than $90 \%$ whatever the number of channels.

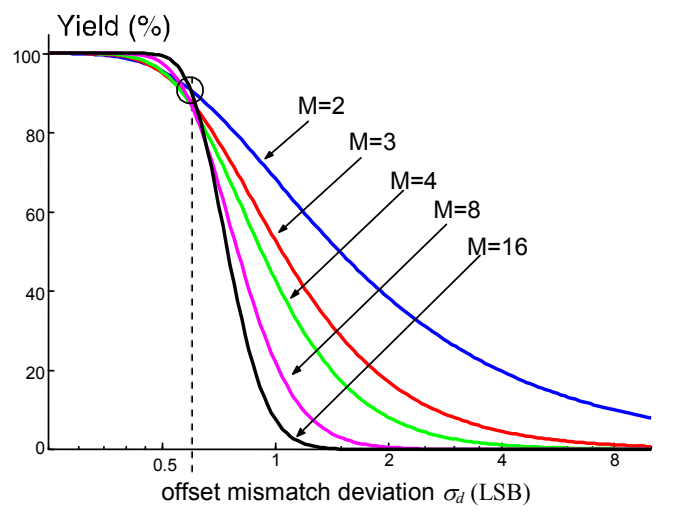

Fig. 14. Yield evolution with offset mismatch, for a 10-bit ADC

The information on the SNR and SFDR should thus be considered in early phases of the design. Thanks to the model, if a designer is able to estimate the expected offset and gain error for a given channel ADC and the expected time-skews associated with a given clocking scheme, he can assess the expected yield of an $M$-time interleaved architecture. If the yield of the planned architecture is too low, he has to evaluate the cost of any corrective action. These corrective actions could be the addition of a calibration scheme, an architecture change or even a technology change. Let us take a brief example: a designer wants to build a $200 \mathrm{MHz} 12$-bit ADC. He planned to use a 2-channel pipeline architecture but the estimation of the offset variance lead him to a poor yield. One solution could be to add a calibration scheme such as proposed in [4], leading to an important increase of the required digital resources. Another solution could be to build a 4-channel ADC. Each channel ADC would thus have a $50 \mathrm{MHz}$ output rate instead of $100 \mathrm{MHz}$. However, using a clock at $100 \mathrm{MHz}$, the extra phase may be used for offset cancellation purpose. Another possible corrective action could be to share as many blocks as possible between the channels that work in opposite phases. If an M-channel ADC is built in this way, it behaves as if it had $M / 2$ channels from a mismatch point of view. This is 
of particular interest in the case of a 2-channel ADC if the mismatches are reduced in a sufficient amount. Conversely, one can start from the SNR and SFDR desired yield and translate this information in terms of mismatches. Then the designer has to evaluate how these new specifications are best reached. It is still up to the designer to value the different trade-off.

\section{CONCLUSIONS}

Time-interleaving is an appealing technique to improve the analog-to-digital conversion speed. However, this strategy will damage the $\mathrm{ADC}$ resolution unless the channel mismatches are kept small.

In this paper, the impact of the gain and offset mismatches, as well as of the time-skew on the SNR and the SFDR of the resulting $\mathrm{ADC}$ have been studied from a probabilistic point of view. The obtained expressions for the Probability Density Functions provide designers with important information which should help to solve design trade-off, to tailor safer margins or to fix clear goals for developing mismatch calibration strategies. Some of the relevant conclusions that can be drawn from the application of the given models can be summarized as follows. Concerning the SNR, its most probable value remains about the same when varying the number of channels but its dispersion is very high for a small number of channel. Concerning the SFDR, its most probable value increases with the number of channels and is higher for an odd number of channel than for an even number. As for the SNR, its dispersion also exhibits a high value for a small number of channels.

\section{APPENDIX}

\section{A. Noise Power calculation}

Let's sketch the steps to come out with (6) from (5),

$$
N_{x} \propto \frac{1}{M^{2}} \sum_{k=1}^{M-1}\left\|Z_{k}\right\|^{2}=\frac{1}{M^{2}}\left[\sum_{k=0}^{M-1}\left\|Z_{k}\right\|^{2}-\left\|Z_{0}\right\|^{2}\right]
$$

where

$$
Z_{k}=\sum_{m=1}^{M} x_{m} e^{-j k m \frac{2 \pi}{M}} \quad, Z_{0}=\sum_{m=1}^{M} x_{m}=M \cdot\left\langle x_{m}\right\rangle
$$

Using conjugate complexes and the Kronecker's delta $\delta_{n m}$, we will arrive to the sample variance of the set $\left\{x_{m}\right\}$

$$
\begin{aligned}
& N_{x} \propto \frac{1}{M^{2}}\left[\sum_{k=0}^{M-1}\left(Z_{k} \cdot Z_{k}^{*}\right)-M^{2}\left\|\left\langle x_{m}\right\rangle\right\|^{2}\right]=\ldots \\
& =\frac{1}{M^{2}}\left[\sum_{m=1}^{M} \sum_{n=1}^{M} x_{m} x_{n}^{*} \sum_{k=0}^{M-1} e^{-j k(m-n) \frac{2 \pi}{M}}\right]-\left\|\left\langle x_{m}\right\rangle\right\|^{2}=\ldots \\
& =\frac{1}{M^{2}}\left[\sum_{m=1}^{M} \sum_{n=1}^{M} x_{m} x_{n}^{*} \cdot\left(M \delta_{n m}\right)\right]-\left\|\left\langle x_{m}\right\rangle\right\|^{2}=\frac{1}{M} \sum_{m=1}^{M}\left\|x_{m}\right\|^{2}-\left\|\left\langle x_{m}\right\rangle\right\|^{2}=\ldots \\
& =\left\langle\left\|x_{m}\right\|^{2}\right\rangle-\left\|\left\langle x_{m}\right\rangle\right\|^{2}=\left\langle\left\|x_{m}-\left\langle x_{m}\right\rangle\right\|^{2}\right\rangle \\
& \text { If } x_{m} \sim N\left(\mu_{x} ; \sigma_{x}\right) \quad \text { and we use the standardized }
\end{aligned}
$$
variables $x_{m}^{\prime}=\left(x_{m}-\mu_{x}\right) / \sigma_{x}$, we obtain, finally,

$$
N_{x} \propto\left\|\sigma_{x}\right\|^{2} \cdot\left\langle\left(x_{m}^{\prime}-\left\langle x_{m}^{\prime}\right\rangle\right)^{2}\right\rangle=\left\|\sigma_{x}\right\|^{2} \cdot \frac{M-1}{M} S_{x}
$$

\section{B. PDF for the power density of an spur}

In order to calculate the probability of a spur power, we have to find the PDF of the variable

$$
S_{x}(k)=\left\|\sum_{m=1}^{M} x_{m}^{\prime} e^{-j \varphi_{k m}}\right\|^{2} \quad, k \in[1,\lfloor M / 2\rfloor]
$$

with $\varphi_{k m}=k m(2 \pi / M)$. The relationship between

We can easily write

$$
\begin{aligned}
S_{x}(k) & =\left\|\left(\sum_{m=1}^{M} x_{m}^{\prime} \cos \varphi_{k m}\right)+j \cdot\left(\sum_{m=1}^{M} x_{m}^{\prime} \sin \varphi_{k m}\right)\right\|^{2}=\ldots \\
& =\left[\left(\sum_{m=1}^{M} x_{m}^{\prime} \cos \varphi_{k m}\right)^{2}+\left(\sum_{m=1}^{M} x_{m}^{\prime} \sin \varphi_{k m}\right)^{2}\right]=\ldots \\
& =\left(S X_{x, k}^{2}+S Y_{x, k}^{2}\right)
\end{aligned}
$$

The last quadratic term is zero if $k=M / 2$ when $M$ is even (this case we will denote hereinafter only with $k=M / 2$ ).

The random variables $S X_{x, k}$ and $S Y_{x, k}$ of (39) are independent. They are projections of a Normal variable over two orthogonal dimensions (the real and imaginary axis) [13].

As each mismatch $x_{m}^{\prime}$ is Normal $N(0 ; 1)$, then the above linear combinations, $S X_{x, k}$ and $S Y_{x, k}$, are also Normal distributions,

$$
\left\{\begin{aligned}
S X_{x, k}=\sum_{m=1}^{M} x_{m}^{\prime} \cos \varphi_{k m} & \sim N\left(0 ; \sqrt{\sum_{m=1}^{M} \cos ^{2} \varphi_{k m}}\right) \\
S Y_{x, k}=\sum_{m=1}^{M} x_{m}^{\prime} \sin \varphi_{k m} & \sim N\left(0 ; \sqrt{\sum_{m=1}^{M} \sin ^{2} \varphi_{k m}}\right)
\end{aligned}\right.
$$

The variances are

$$
\begin{aligned}
& \sum_{m=1}^{M} \cos ^{2} \varphi_{k m}=\frac{1}{2} \sum_{m=1}^{M}\left(1+\cos 2 \varphi_{k m}\right)=\frac{1}{2} \sum_{m=1}^{M}\left(1+\operatorname{Re}\left(e^{j 2 \varphi_{k m}}\right)\right)=\ldots \\
& = \begin{cases}M / 2 & \text {,if } k \neq M / 2 \\
M & \text {,if } k=M / 2\end{cases} \\
& \sum_{m=1}^{M} \sin ^{2} \varphi_{k m}=\frac{1}{2} \sum_{m=1}^{M}\left(1-\cos 2 \varphi_{k m}\right)=\frac{1}{2} \sum_{m=1}^{M}\left(1-\operatorname{Re}\left(e^{j 2 \varphi_{k m}}\right)\right)=\ldots \\
& = \begin{cases}M / 2 & \text {,if } k \neq M / 2 \\
0 & \text {,if } k=M / 2\end{cases}
\end{aligned}
$$

If $\left.S_{x}(k)\right|_{k \neq M / 2}$ is the sum of two independent squared normal variables then it behaves like a Chi-square law with two degrees of freedom $\chi_{2}^{2}$, and when $k=M / 2$ is distributed as the square of a normal variable, like a $\chi_{1}^{2}$.

\section{Statistical independence between spur powers}

To demonstrate that two spur powers, $P_{x}^{\text {spur }}(k), P_{x}^{\text {spur }}(l)$ with $k, l \in\{1, \ldots,\lfloor\mathrm{M} / 2\rfloor\}$ are statistically independents, we should keep in mind that the relationship between the total noise power $N_{x}$ and the individual power of each spur $P_{x}^{s p u r}(k)$ is 


$$
N_{x}=\sum_{k=1}^{M-1} P_{x}^{\text {spur }}(k)
$$

or the same using the definitions (8) and (38),

$$
s_{x}=\frac{1}{M(M-1)} \sum_{k=1}^{M-1} S_{x}(k) \text {. }
$$

Developing (44) we have an expression that verify the conditions of Cochran's theorem [14],

$$
\begin{aligned}
& M \sum_{m=1}^{M} x_{m}^{\prime 2}=M^{2}\left\langle x_{m}^{\prime}\right\rangle^{2}+2 \sum_{k=1}^{\lfloor M / 2\rfloor}\left[S X_{x, k}^{2}+S Y_{x, k}^{2}\right]=\ldots \\
& =\left(\sum_{m=1}^{M} x_{m}^{\prime}\right)^{2}+\sum_{k=1}^{\lfloor M / 2\rfloor} 2 \cdot\left[\left(\sum_{m=1}^{M} x_{m}^{\prime} \cos \varphi_{k m}\right)^{2}+\left(\sum_{m=1}^{M} x_{m}^{\prime} \sin \varphi_{k m}\right)^{2}\right]
\end{aligned}
$$

This is a very important theorem which allows us to decompose sums of squares into several quadratic forms and identify their distributions to Chi-square $\chi_{v}^{2}$ and establish their mutual independence. Under these conditions the quadratic forms $\left\{S_{x}(k)\right\}, k \in\{1, \ldots\lfloor M / 2\rfloor\}$ are statistically independent and therefore, the spur powers are also independent.

\section{REFERENCES}

[1] M. Choi, A.A. Abidi, "A 6-b 1.3-Gsample/s A/D converter in $0.35-\mu \mathrm{m}$ CMOS," IEEE-Journal-of-Solid-State-Circuits. vol. 36, no. 12, pp. 1847-1858, Dec. 2001.

[2] R.H. Walden, "Analog-to-digital converter survey and analysis," IEEE Journal-on-Selected-Areas-in-Communications, vol. 17, no. 4, April 1999.

[3] W.C. Black-Jr, D.A. Hodges, "Time interleaved converter arrays," IEEE Journal-of-Solid-State-Circuits. vol. SC-15, no. 6, pp.1022-1029, Dec. 1980.

[4] N. Kurosawa, H. Kobayashi, K. Kobayashi, "Channel linearity mismatch effects in time-interleaved ADC systems," in Proc. of ISCAS 2001, pp. 420-423, vol. 1, 2001.

[5] Y.C. Jenq, "Digital Spectra of Nonuniformly Sampled Signals: Fundamentals and High-Speed Waveform Digitizers," IEEE Trans. on Instrumentation and Measurements, vol. 37, no. 2, pp. 245-251, June 1997.

[6] M. Gustavsson, J.J. Wilkner, N.N. Tan, CMOS Data Converters for Communications, Kluwer Academic Publishers, 2000.

[7] N. Kurosawa, H. Kobayashi, K. Maruyama, H. Sugawara, K. Kobayashi, "Explicit analysis of channel mismatch effects in timeinterleaved ADC systems," IEEE Trans. on Circuits and Systems I: Fundamental Theory and Applications. vol. 48, no. 3, pp. 261-271, March 2001.

[8] A. Petraglia-A, S.K. Mitra, "Analysis of mismatch effects among A/D converters in a time-interleaved waveform digitizer," IEEE Trans. on Instrumentation and Measurement, vol. 40, no. 5, pp. 831-835, October 1991.

[9] J.E. Eklund, F. Gustafsson, "Digital offset compensation of timeinterleaved ADC using random chopper sampling," in Proc. of ISCAS 2000, vol. 3, pp. 447-450, 2000.

[10] Daihong-Fu, K.C. Dyer, S.H. Lewis, P.J. Hurst, "A digital background calibration technique for time-interleaved analog-to-digital converters," IEEE Journal-of-Solid-State-Circuits, vol. 33, no. 12, pp. 1904-1911, Dec. 1998.

[11] Y.C. Jenq, "Perfect Reconstruction of Digital Spectrum from Nonuniformly Sampled Signals," IEEE Trans. on Instrumentation and Measurements, vol. 46, no. 3, pp. 649-652, June 1997.

[12] G. Leger, E. Peralías, A. Rueda, "SNR probability in time-interleaved ADCs with random channel mismatches," in Proc. of ISCAS 2002, vol. 2, pp. 380-383, 2002.

[13] A. Papoulis, Probability, Random Variables, and Stochastic Processes, McGRAW-HILL International Editions, third edition, 1991.

[14] R.V. Hogg and A.T. Craig, Introduction to Mathematical Statistics, 5th edition, 1989, Macmillan. 